\title{
Procedimiento para el análisis de vibración inducida por flujo turbulento en tuberías usando simulación numérica
}

\author{
Rosendo Franco(1), Michael A. Blas ${ }^{(1)}$, Leonidas Rodríguez ${ }^{(1)}$ y L. Enrique Ortiz-Vidal(2) \\ (1) Grupo INACOM/Aula PUCP-CIMNE, Sección Ing. Mecánica, Dpto. de Ingeniería, Pontificia Universidad Católica del \\ Perú, Lima-Perú. (correo-e: rofranco@pucp.pe; michael.blas@pucp.pe; leonidas.rodriguez@pucp.pe) \\ (2) Instituto de Ciencias de la Ingeniería, Universidad de O'Higgins (UOH), Av. Libertador Bernardo O'Higgins 611, \\ Rancagua-Chile. (correo-e: enrique.ortiz@uoh.cl)
}

Recibido Ene. 2, 2020; Aceptado Mar. 5, 2020; Versión final Mar. 22, 2020, Publicado Jun. 2020

\begin{abstract}
Resumen
Se propone un procedimiento para estudiar el fenómeno de vibración inducida por flujo (FIV) en tuberías que transportan fluido newtoniano en régimen turbulento. Se usa un modelo numérico que considera la interacción fluido-estructura a través del acoplamiento bidireccional entre estos dominios físicos. Se emplean los métodos de Volúmenes Finitos y Elementos Finitos para resolver los dominios fluidodinámico y estructural, respectivamente. Se considera: (i) condición de periodicidad para obtener rápidamente un flujo turbulento totalmente desarrollado, (ii) modelo WMLES S-Omega para la turbulencia, (iii) tubería de material elástico lineal, y (iv) teoría de grandes desplazamientos. El modelo se implementa en el software ANSYS 2019 R1. Se simulan tres condiciones de flujo de agua en una tubería horizontal biempotrada de PVC de $20.4 \mathrm{~mm}$ de diámetro interno. Los resultados muestran los mismos efectos de la velocidad sobre la amplitud y frecuencia de vibración que los reportados en estudios experimentales y analíticos, validando el procedimiento propuesto.
\end{abstract}

\section{Procedure for turbulent flow-induced vibration analysis of pipes using numerical simulation}

\begin{abstract}
A procedure for the study of the flow-induced vibration (FIV) phenomenon of pipes conveying turbulent Newtonian fluid flow is proposed. A numerical model that considers the fluid-structure interaction through the bidirectional coupling between these physical domains is used. The Finite Volume and Finite Element methods, respectively, are used to solve the fluid-dynamic and structural domains. The model considers: (i) periodicity condition for quickly obtaining a fully developed turbulent flow, (ii) WMLES S Omega model for turbulence, (iii) pipe with linear elastic material, and (iv) large displacements theory. The model is implemented in ANSYS 2019 R1 software. Three water flow conditions in a clamped-clamped horizontal PVC pipe with $20.4 \mathrm{~mm}$ internal diameter are simulated. The results show the same velocity effects on the amplitude and frequency vibration reported by experimental and analytical studies, validating the proposed procedure.
\end{abstract}

Keywords: turbulent pipe flow; WMLES S-Omega; flow-induced vibration; fluid-structure interaction 


\section{INTRODUCCIÓN}

El estudio del fenómeno de Vibración Inducida por Flujo (FIV, del inglés Flow Induced Vibration) en tuberías se inició intensamente en la década de los 50, entorno a los problemas que se presentaron en uno de los oleoductos de la Trans-Arabian Pipe Line Company (Ashley et al., 1950). En este fenómeno, el flujo genera fuerzas dinámicas sobre la tubería, dando lugar a la vibración de la misma, pudiendo provocar incluso la falla estructural. Dada la fuerte presencia del transporte de fluidos en tuberías en distintos sectores industriales, como petroquímico, nuclear, petróleo y gas, el estudio detallado del fenómeno FIV es relevante.

En vibraciones mecánicas la amplitud y la frecuencia de vibración son dos parámetros importantes para analizar la respuesta dinámica de un sistema. Estudios FIV en tuberías han reportado que ambos parámetros varían con la velocidad del flujo. Por ejemplo, para tuberías sujetas en ambos extremos, la amplitud de vibración incrementa con la velocidad del flujo (Evans et al., 2004, Païdoussis, 1998). De manera opuesta, la frecuencia de vibración disminuye con el aumento de la velocidad (Païdoussis, 1998). Si la velocidad del flujo es tan alta que la frecuencia de vibración llega a cero, se produce la pérdida de estabilidad y la estructura colapsa (Dodds et al., 1965). El fenómeno FIV en tuberías ha sido ampliamente estudiado mediante modelos unidimensionales. Sin embargo, son pocos los estudios tridimensionales reportados, principalmente, debido al alto costo computacional que representan los métodos numéricos utilizados para estos fines. El desarrollo actual de hardware ha permitido que se hayan realizado algunos estudios tridimensionales, utilizando el enfoque conocido como Interacción Fluido-Estructura (FSI, del inglés Fluid-Structure Interaction). En este enfoque se utiliza el Método de Volúmenes Finitos (FVM) para resolver el dominio fluidodinámico y el Método de Elementos Finitos (FEM) para resolver el dominio estructural. La interacción entre ambos dominios puede modelarse mediante un acoplamiento unidireccional o bidireccional. En el acoplamiento unidireccional solo se transmiten los efectos del fluido sobre la estructura, mientras que, en el bidireccional se consideran además los efectos de la estructura sobre el fluido. Para resolver problemas que involucran la vibración estructural es adecuado usar el acoplamiento bidireccional (Zienkiewicz et al., 2014).

Desde el punto de vista tridimensional, Pittard y Blotter (2003) modelaron la vibración inducida por flujo turbulento en tubería usando un acoplamiento unidireccional. Para el flujo turbulento usaron FVM, junto a un modelo de turbulencia LES (del inglés Large Eddy Simulation), y para la estructura usaron FEM. Los autores correlacionaron la aceleración estructural con la velocidad del flujo. Shurtz et al. (2010), con base al modelo numérico propuesto por Pittard y Blotter (2003), realizaron estudios numéricos y obtuvieron expresiones que relacionan desplazamiento, velocidad y aceleración de la tubería con parámetros fluidodinámicos y estructurales. Loh et al. (2013) analizaron la respuesta dinámica de una tubería en U mediante simulación numérica. A diferencia de los trabajos anteriores, los autores consideraron un acoplamiento bidireccional. Los estudios numéricos reportados no son concluyentes con relación a las tendencias experimentales observadas en la amplitud y frecuencia de vibración. Así, son necesarios mayores estudios para confirmar la validez del uso de herramientas numéricas en la investigación de FIV en tuberías.

En este trabajo se propone un procedimiento para estudiar numéricamente el fenómeno FIV en tuberías que transportan fluido newtoniano en régimen turbulento. Se presenta un modelo tridimensional, con enfoque FSI y acoplamiento bidireccional, y una estrategia de simulación capaz de predecir las tendencias experimentales reportadas. El procedimiento se verifica y valida a través del análisis de una tubería con diferentes condiciones de flujo turbulento.

\section{MODELADO NUMÉRICO}

Como se ha indicado, para estudiar la vibración inducida por flujo turbulento en la tubería horizontal, se utiliza un modelo tridimensional, con enfoque FSI y acoplamiento bidireccional. Este enfoque considera las fuerzas producidas por el flujo turbulento -presión y esfuerzo cortante- sobre la pared de la tubería y los desplazamientos de la tubería sobre el fluido. La Fig. 1 muestra un esquema del sistema a analizar, donde $p(t)$ representa el campo de presiones sobre la tubería sin deformar y $p^{*}(t)$ el campo de presiones en un instante posterior, considerando la deformación de la tubería.
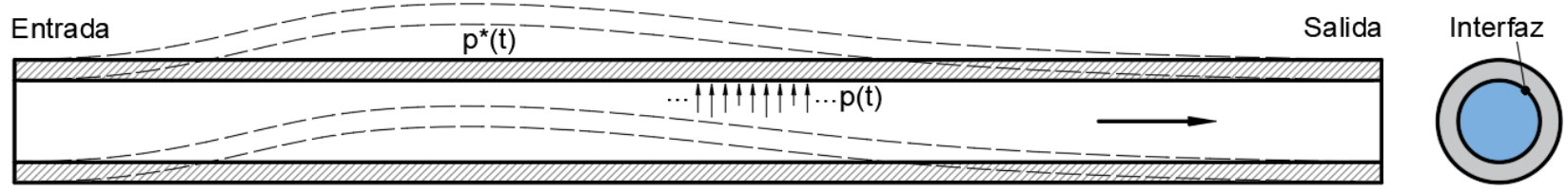

Fig. 1: Tubería sometida a flujo turbulento, sin deformar (línea continua) y deformada (línea de trazos) 
El modelo numérico está estructurado en tres niveles, como se muestra en la Fig. 2. El primer nivel considera las simulaciones de cada dominio físico, i.e. bucles de los dominios fluidodinámico y estructural. En este nivel, se resuelve el dominio fluidodinámico y se calculan las fuerzas sobre la pared de la tubería (la interfaz, ver Fig. 1). Con tales fuerzas se resuelve el dominio estructural para determinar sus desplazamientos. En el segundo nivel se encuentra el bucle de acoplamiento. Este se encarga de verificar que los resultados de fuerzas fluidodinámicas y desplazamientos estructurales alcancen la convergencia. En caso no se logre, se actualiza el dominio fluidodinámico y se repiten las simulaciones del primer nivel. Cuando se alcance la convergencia, se termina el bucle de acoplamiento. El tercer nivel corresponde al bucle de tiempo. Este se encarga de incrementar el tiempo para las simulaciones y termina cuando se alcanza el tiempo de análisis establecido.

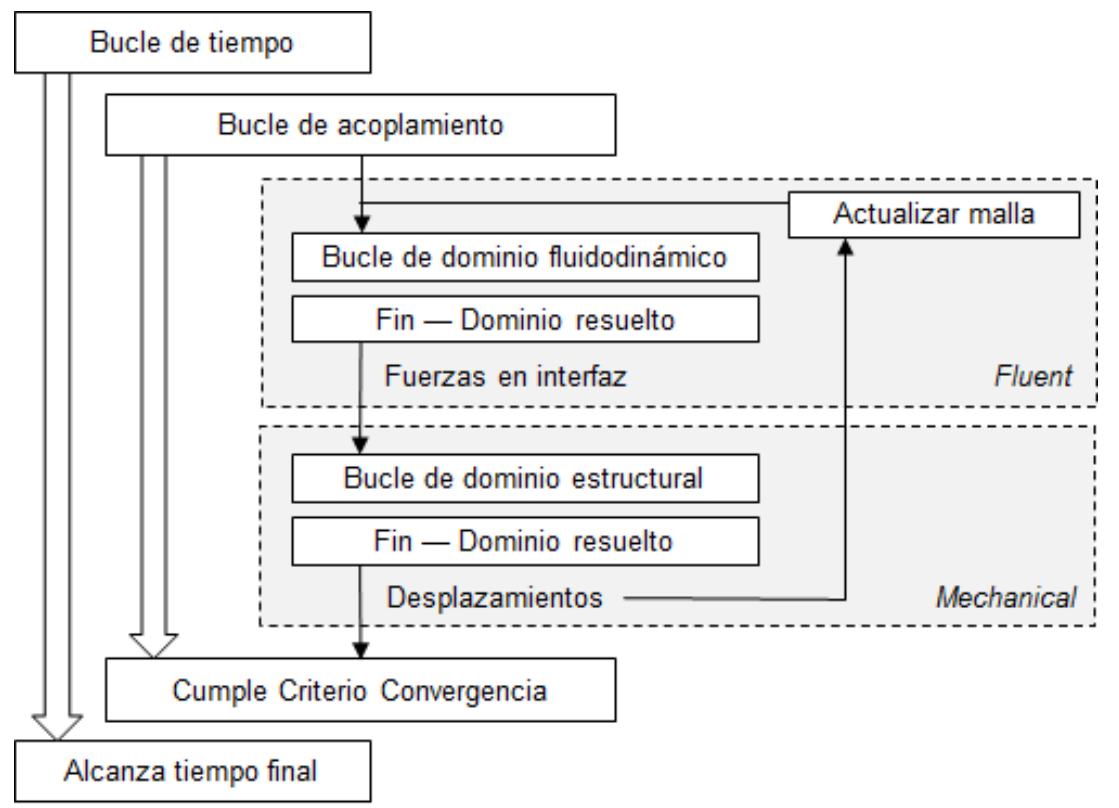

Fig. 2: Esquema del modelo numérico con acoplamiento bidireccional

Para analizar la convergencia del acoplamiento se utiliza el parámetro de control $\phi$, definido por la ecuación (1), donde $a_{\text {new }}$ y $a_{\text {old }}$ son la fuerza o el desplazamiento calculado en la última y penúltima iteración, respectivamente. Las sumatorias consideran a todos los nodos ubicados en la interfaz (ANSYS, 2018). En el presente estudio se establece $\phi \leq 0.01$. El mismo criterio de convergencia ha sido utilizado por Liu et al. (2018) y Pei et al. (2012).

$\phi=\frac{\sqrt{\sum\left(\mathrm{a}_{\text {new }}-\mathrm{a}_{\text {old }}\right)^{2}}}{\sqrt{\sum \mathrm{a}_{\text {new }}^{2}}}$

\section{Modelado del flujo}

Las ecuaciones de transporte son discretizadas y resueltas usando FVM. Se considera un fluido newtoniano y flujo turbulento e incompresible. La turbulencia se modela usando WMLES S-Omega. Se trata de un modelo de la familia LES. En general, las técnicas LES resuelven las estructuras turbulentas "grandes" y modelan las "pequeñas". En particular, WMLES S-Omega tiene ventajas frente a otras técnicas LES, pues contempla un modelo de pared adecuado para simular la turbulencia en dicha zona sin requerir una discretización extremadamente fina (Park y Moin, 2016; Sayadi y Moin, 2016).

El modelado numérico del flujo se realiza con una condición de periodicidad en la dirección del flujo (Eggels, 1994; Peng et al., 2018; Wu y Moin, 2008). Esta condición es utilizada para estudiar flujo turbulento en tuberías, sin necesidad de considerar la longitud de desarrollo total. De esta manera, se reduce el costo computacional para alcanzar el régimen turbulento totalmente desarrollado. Asimismo, se considera la tubería lisa (sin rugosidad) y la condición de no deslizamiento en la pared. La aplicación de la condición de periodicidad exige el conocimiento del gradiente de presión en la dirección del flujo, $\mathrm{dP} / \mathrm{dz}$. Para flujo en tubería horizontal este gradiente es causado por la fricción y puede ser obtenido usando el método de DarcyWeisbach (Cengel y Cimbala, 2018; Ortiz-Vidal et al., 2010), según la ecuación (2), donde $\rho, v, d$ y $\xi$ 
representan la densidad del fluido, la velocidad media del flujo, el diámetro interno de la tubería y el factor de fricción respectivamente.

$$
\frac{d P}{d z}=\frac{1}{2} \xi \frac{\rho v^{2}}{d}
$$

El factor de fricción se puede estimar usando la correlación de Churchill (Cengel y Cimbala, 2018), como se indica en las expresiones (3), donde e representa la rugosidad absoluta de la tubería.

$$
\begin{aligned}
& \xi=8\left[\left(\frac{8}{\operatorname{Re}}\right)^{12}+\frac{1}{(\mathrm{~A}+\mathrm{B})^{3 / 2}}\right]^{1 / 12} \\
& \mathrm{~A}=\left\{-2.457 \ln \left[\left(\frac{7}{\operatorname{Re}}\right)^{0.9}+0.27 \frac{\mathrm{e}}{\mathrm{d}}\right]\right\}^{16} \\
& \mathrm{~B}=\left(\frac{37530}{\operatorname{Re}}\right)^{16}
\end{aligned}
$$

El parámetro Re representa el número de Reynolds y para flujo en tuberías se define por la ecuación (4), donde $\mu$ es la viscosidad dinámica del fluido.

$$
\mathrm{Re}=\frac{\rho \mathrm{vd}}{\mu}
$$

En la literatura existen numerosas correlaciones para el cálculo del factor de fricción. Sin embargo, se recomienda la correlación de Churchill pues es válida para todos los regímenes de flujo, y entrega simplicidad y adecuada precisión (Cengel y Cimbala, 2018; Ortiz-Vidal et al., 2010). El dominio geométrico del fluido se discretiza usando una malla estructurada tipo mariposa. Esta consta de una discretización circular en la zona cerca a la pared y una rectangular en la zona central (ver Fig. 3). Esta discretización permite afinar la malla en la zona cercana a la pared sin necesidad de hacerla al centro. Este tipo de malla es usada en estudios de flujos turbulentos en tuberías, ya que se obtiene una buena calidad de malla (El Khoury et al.,2013; Hernandez-Perez et al., 2011).

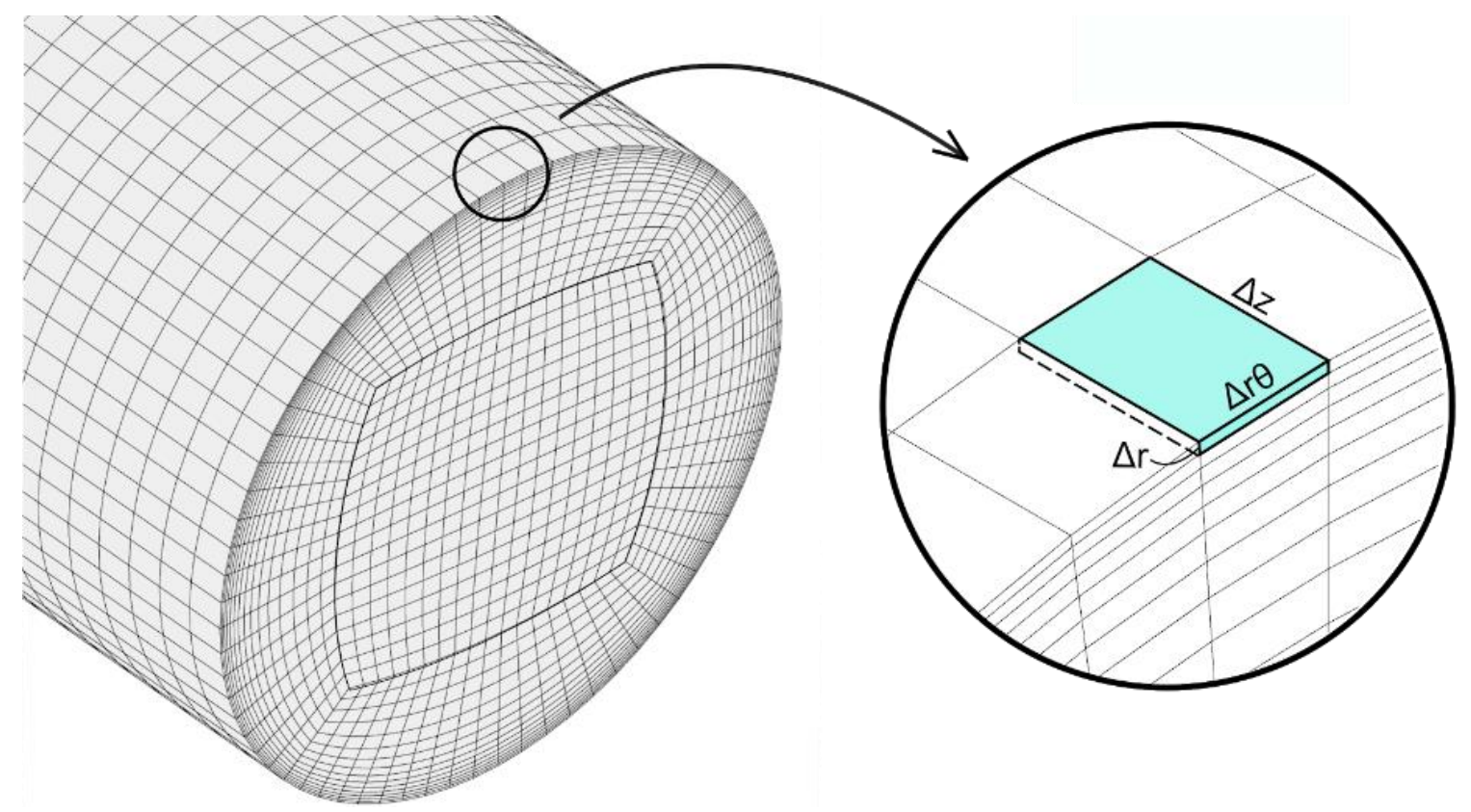

Fig. 3: Discretización del dominio fluidodinámico para una malla genérica tipo mariposa

El tamaño de las celdas en la pared en las direcciones radial $\Delta r$, circunferencial $\Delta r \theta$ y axial $\Delta z$, se determina en función de los parámetros adimensionales $r^{+}, r \theta^{+}$y $z^{+}$, respectivamente. La literatura recomienda los valores $\mathrm{r}^{+}=1, r \theta^{+}=20 \mathrm{y} \mathrm{z}^{+}=40$ (Piomelli, 1997; Shurtz et al.,2010). El tamaño de la celda en cada dirección se obtiene reemplazando los valores recomendados en la ecuación (5), donde $v_{f}$ representa la velocidad de fricción, que es función del esfuerzo cortante en la pared $\mathrm{T}_{\mathrm{w}}$ (Cengel y Cimbala, 2018). 
Tamaño de celda $=$ Parámetro adimensional $\cdot\left(\frac{\mu}{\rho \cdot v_{f}}\right)$

A partir de un análisis de volumen de control es posible relacionar el esfuerzo cortante en la pared con el gradiente de presión por fricción, y finalmente expresar la velocidad de fricción en términos de la velocidad media y del factor de fricción, como se indica en la ecuación (6).

$$
v_{f}=\sqrt{\frac{T_{w}}{\rho}}=v \sqrt{\frac{\xi}{8}}
$$

Con relación al tamaño de celdas, para disminuir el costo computacional se recomienda un incremento geométrico de 1.2 para el $\Delta \mathrm{r}$, partiendo de la pared. El tamaño de malla en la dirección axial permanece constante. El criterio para establecer el paso de tiempo de la simulación se basa en el número de Courant, definido por la expresión (7), donde u representa la velocidad local en la dirección del flujo, $\Delta t$ es el paso de tiempo y $\Delta x$ es el tamaño de celda.

$$
c=\frac{u \Delta t}{\Delta x}
$$

En la dinámica de fluidos computacional, el paso de tiempo debe capturar los tiempos característicos convectivos y difusivos de las escalas a resolver. En tal sentido, la literatura especializada señala que las simulaciones deben considerar un número de Courant menor a uno (Fernández, 2010; Ferziger y Peric, 2002).

\section{Modelado estructural}

Las ecuaciones de equilibrio dinámico son discretizadas y resueltas empleando FEM. Para la integración temporal de la ecuación discretizada se utiliza el método de Newmark (Newmark, 1959). Se consideran la teoría de grandes desplazamientos y un modelo elástico lineal para el material de la tubería. Como condición de frontera, se restringen completamente los desplazamientos en las caras anulares de los extremos de la tubería (tubería biempotrada). La discretización del dominio geométrico estructural se basa en una malla estructurada empleando elementos hexaédricos de primer orden (8 nodos). En las direcciones axial y circunferencial el tamaño del elemento es el doble del considerado en el dominio fluidodinámico. En la dirección radial de la tubería (espesor) se utilizan dos elementos (ver Fig. 4). Por su parte, el paso de tiempo considerado para resolver el dominio estructural es el mismo que el utilizado para resolver el dominio fluidodinámico.

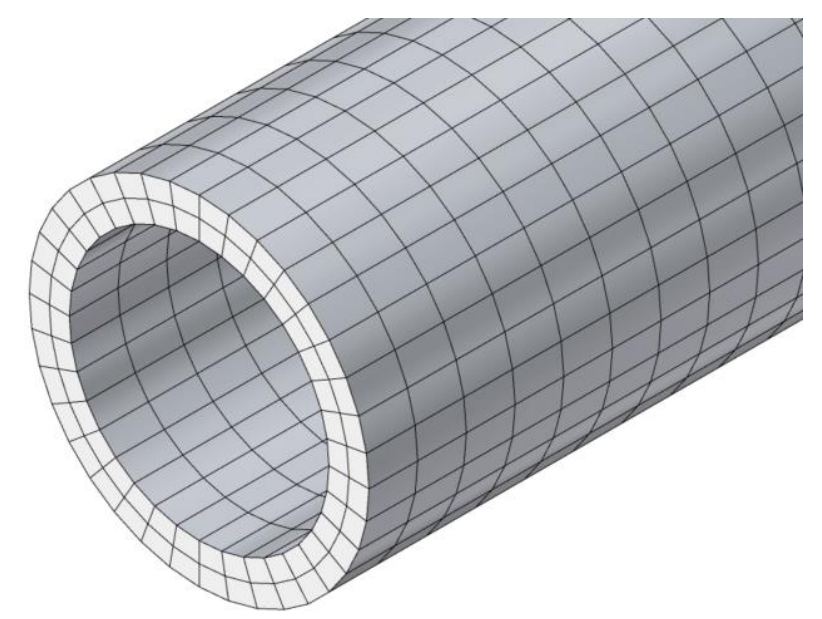

Fig. 4: Discretización del dominio estructural para una malla genérica

\section{Implementación Numérica y Estrategia de Simulación}

El modelo numérico descrito se implementa a través de la plataforma ANSYS Workbench 2019 R1. EI acoplamiento bidireccional se realiza mediante el componente System Coupling. Se usan los sistemas de análisis de fluidos (Fluent) y análisis estructural transitorio (Mechanical) para resolver los dominios fluidodinámico y estructural, respectivamente. Asimismo, se usa ICEM CFD y Meshing para la respectiva discretización de dichos dominios. Como se mencionó, para garantizar el acoplamiento bidireccional, en cada 
iteración debe actualizarse la discretización del dominio fluidodinámico, en función de los desplazamientos de la estructura. Esto se logra mediante la opción Dynamic Mesh en Fluent. El algoritmo de actualización de malla elegido es Smoothing-Diffusion (ANSYS, 2018). Para el análisis del fluido, el esquema de acoplamiento velocidad-presión es resuelto usando el algoritmo SIMPLE, junto a un esquema de solución transitorio basado en la presión. La formulación empleada para discretizar el término transitorio es First Order Implicit. El esquema empleado para la discretización del gradiente es Least Squares Cell Based. El esquema para la discretización de la presión es Second Order. El esquema para la discretización del momento lineal es Bounded Central Differencing. Estos esquemas son recomendados para resolver problemas de turbulencia transitorios (ANSYS, 2018).

La estrategia de simulación consta de dos etapas. En la primera etapa se resuelve el flujo de manera independiente, hasta que alcanza un régimen turbulento totalmente desarrollado. Para esto se usa una condición de periodicidad traslacional, que consiste en definir el gradiente de presión entre la entrada y la salida de la tubería. Esta condición de contorno es muy conveniente para reducir el costo computacional, ya que se analiza sólo la longitud de estudio de la tubería (longitud entre apoyos), evitando analizar adicionalmente la longitud requerida para el desarrollo natural del flujo. El flujo totalmente desarrollado se alcanza cuando el esfuerzo cortante promedio se hace constante en el tiempo (Cengel y Cimbala, 2018). En la segunda etapa se realiza el análisis acoplado FSI, según el procedimiento descrito. Como condición inicial del dominio fluidodinámico se toman los últimos resultados de la primera etapa (flujo desarrollado).

\section{RESULTADOS Y DISCUSIÓN}

Se analiza la vibración inducida por flujo interno turbulento de una tubería horizontal biempotrada, con diámetro interno $0.0204 \mathrm{~m}$, diámetro externo $0.0267 \mathrm{~m}$, longitud entre apoyos $1.53 \mathrm{~m}$, material PVC (módulo de Young 2.4 GPa y coeficiente de Poisson 0.4). Para el fluido se considera agua con densidad $998.2 \mathrm{~kg} / \mathrm{m}^{3}$ y viscosidad dinámica 0.001003 Pa.s. Los resultados presentados son para la sección media de la tubería.

\section{Parámetros para la simulación}

La Tabla 1 muestra las condiciones de flujo evaluadas. Se considera que un número de Reynolds mayor a 4000 garantiza flujo turbulento (Cengel y Cimbala, 2006). El gradiente de presión requerido por la condición de periodicidad se calculó usando el método de Darcy-Weisbach con la correlación de Churchill para el factor de fricción, como fue descrito en la sección de modelado del flujo.

Tabla 1: Condiciones de flujo simuladas y gradiente de presión teórica

\begin{tabular}{|c|c|c|c|}
\hline Caso & Velocidad media, $\mathrm{v}(\mathrm{m} / \mathrm{s})$ & Número de Reynolds, Re & Gradiente de presión teórica, $\mathrm{dP} / \mathrm{dz}(\mathrm{Pa} / \mathrm{m})$ \\
\hline 1 & 0.25 & 5300 & 62.04 \\
\hline 2 & 0.50 & 10150 & 188.84 \\
\hline 3 & 0.75 & 15225 & 423.20 \\
\hline
\end{tabular}

Parámetros de la discretización de los dominios fluidodinámico y estructural se muestran en la Tabla 2. Los tamaños de las celdas se determinaron empleando la ecuación (5) y los valores recomendados para el tamaño adimensional de celda. La velocidad de fricción se calculó usando las ecuaciones (3), (4) y (6). El paso de tiempo $(\Delta t)$ se estimó mediante la ecuación (7), considerando un número de Courant igual a 1 y la velocidad máxima en el centro de la tubería (1.3 veces la velocidad media). El paso del tiempo es el mismo para ambos dominios. En todos los casos se considera un tiempo de análisis acoplado FSI igual a $4 \mathrm{~s}$.

Tabla 2: Parámetros de la discretización (tamaño de celdas, paso de tiempo, número de celdas y de elementos)

\begin{tabular}{|c|c|c|c|c|c|c|}
\hline Caso & $\Delta \mathrm{r}(\mathrm{m})($ pared $)$ & $\Delta \mathrm{r} \theta(\mathrm{m})$ & $\Delta \mathrm{z}(\mathrm{m})$ & $\begin{array}{c}\text { Paso de } \\
\text { tiempo }(\mathrm{s})\end{array}$ & $\begin{array}{c}\text { Número de celdas } \\
- \text { Flujo }\end{array}$ & $\begin{array}{c}\text { Número de elementos } \\
\text { Estructura }\end{array}$ \\
\hline 1 & $5.1 \times 10^{-5}$ & $8.9 \times 10^{-4}$ & $1.91 \times 10^{-3}$ & 0.0024 & 1123200 & 28800 \\
\hline 2 & $3.24 \times 10^{-5}$ & $7.25 \times 10^{-4}$ & $1.51 \times 10^{-3}$ & 0.0011 & 2068000 & 36000 \\
\hline 3 & $2.28 \times 10^{-5}$ & $6.65 \times 10^{-4}$ & $1.27 \times 10^{-3}$ & 0.0006 & 2995200 & 57600 \\
\hline
\end{tabular}

\section{Comportamiento Fluidodinámico}

La Fig. 5 muestra el valor del esfuerzo cortante promedio en la pared de la tubería, en función del tiempo, para las tres condiciones de flujo. Se aprecia que se requiere de cierto tiempo de simulación para que los valores de esfuerzo cortante se estabilicen, alcanzando un valor constante. Este es el indicador de un flujo turbulento totalmente desarrollado, como se mencionó en la sección de modelado numérico. Los valores 
medios varían para cada caso de estudio. Por ejemplo, para el primer caso el valor tiende a estabilizarse en $0.295 \mathrm{~Pa}$, para el segundo caso en 0.971 $\mathrm{Pa}$ y para el tercer caso en $2.020 \mathrm{~Pa}$.

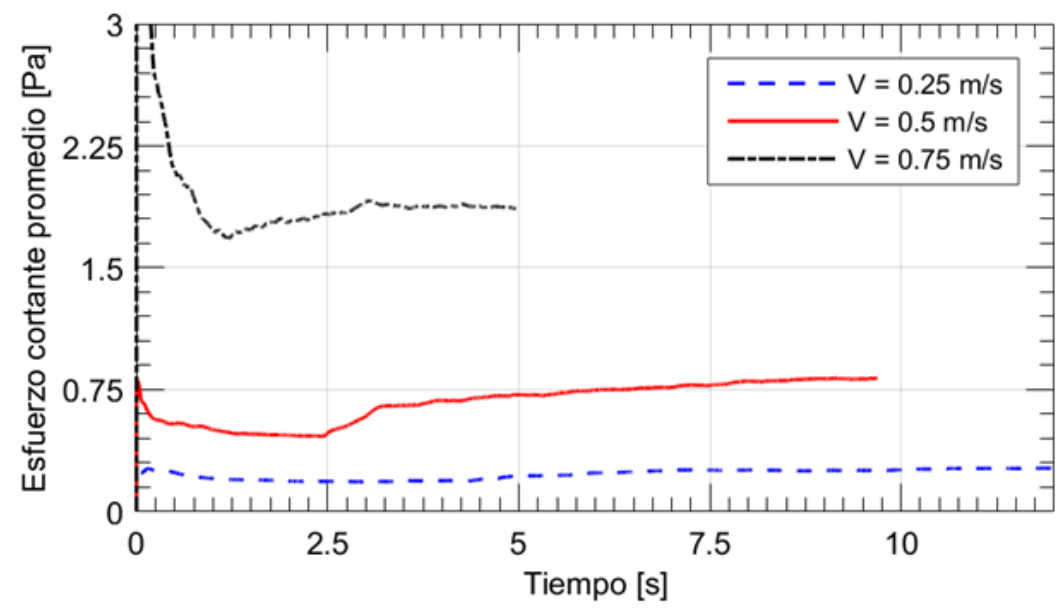

Fig. 5: Evolución del esfuerzo cortante promedio en la pared de la tubería

La Fig. 6 muestra los perfiles de velocidad para las tres condiciones de flujo. El eje de las ordenadas se presenta normalizado, mostrando el cociente de la velocidad promedio para la posición radial específica y la velocidad media del flujo. La Fig. 6 también muestra resultados teóricos de la ley de potencia (Fox et al., 2003). Al comparar las simulaciones con los resultados teóricos se muestra una adecuada concordancia, principalmente para valores de $r / R$ entre 0 y 0.8 . Para valores de $r / R$ entre 0.8 y 1 la diferencia entre los resultados es apreciable. Esta diferencia disminuye conforme aumenta la velocidad de flujo, y por ende el número de Reynolds. Esta tendencia permite inferir que la diferencia entre resultados numéricos y teóricos es una función del nivel de turbulencia, cuantificado por el número de Reynolds. Resultados similares son encontrados en la literatura (Wagner et al., 2001). Para el caso específico de la velocidad máxima $(r / R=0$, i.e. en el centro de la tubería), estudios teóricos y numéricos reportan un cociente de 1.3 aproximadamente (Peng et al., 2018; White, 2011). En el presente trabajo, se tienen cocientes de 1.36, 1.32 y 1.24 para las condiciones de $0.25 \mathrm{~m} / \mathrm{s}, 0.5 \mathrm{~m} / \mathrm{s}$ y $0.75 \mathrm{~m} / \mathrm{s}$, respectivamente.

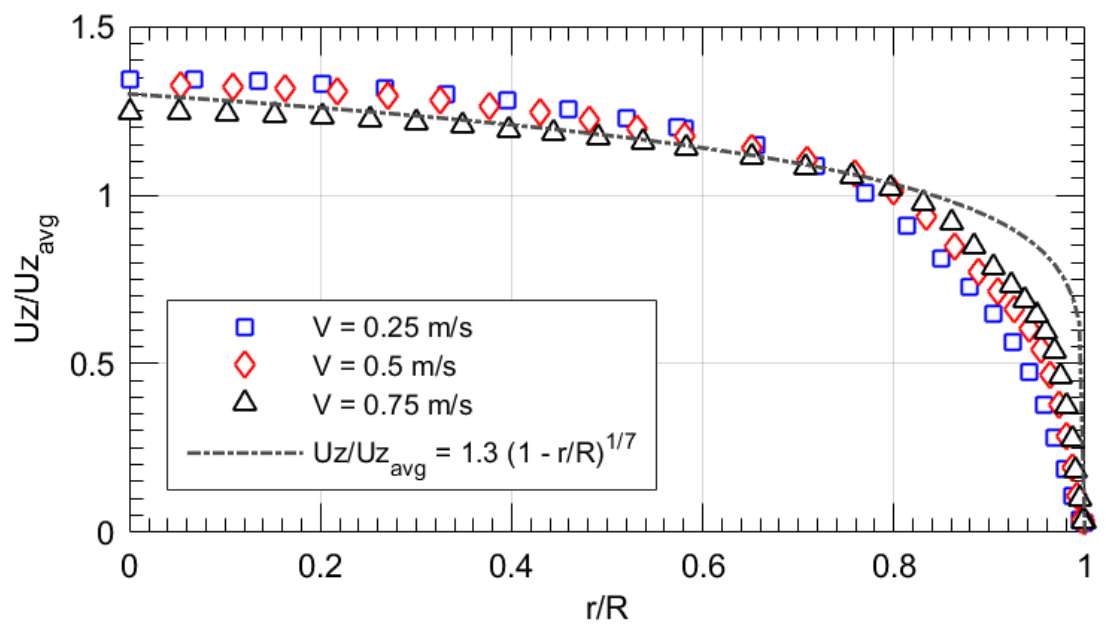

Fig. 6: Perfil de velocidad en el radio de la tubería, numérico (con marcadores) y ley de potencia (línea de trazos)

La Fig. 7 muestra los perfiles de velocidad en términos de los parámetros adimensionales $\mathrm{y}^{+} \mathrm{y} \mathrm{u}^{+}$en la región de la capa límite, para los tres casos. Se muestra también las expresiones empíricas conocidas como ley de pared (línea de trazo y punto) y ley de capa logarítmica (línea de trazo), reportado por Fox et al. (2003). Como se observa, en la subcapa laminar viscosa $\left(0<\mathrm{y}^{+}<5\right)$, los resultados numéricos tienen una adecuada concordancia con la relación establecida $\mathrm{u}^{+}=\mathrm{y}^{+}$. Sin embargo, los resultados numéricos en la región logarítmica $\left(\mathrm{y}^{+}>30\right)$ difieren en cierta medida de la teoría (que en realidad es un ajuste de resultados empíricos). Se observa que esta diferencia disminuye con el aumento de la velocidad, presentando la misma tendencia que el cociente de la velocidad. Cuantitativamente, para el rango $\left(30<\mathrm{y}^{+}<100\right)$, la diferencia relativa varía entre $15 \%$ y $36 \%$ para $0.25 \mathrm{~m} / \mathrm{s}$; entre $6 \%$ y $34 \%$ para $0.5 \mathrm{~m} / \mathrm{s}$; y entre $2.5 \%$ y $24 \%$ para $0.75 \mathrm{~m} / \mathrm{s}$. Esta tendencia también se observa en otros trabajos (Eggels et al., 1994; El Koury et al., 2013 y Wagner et al., 2001). 


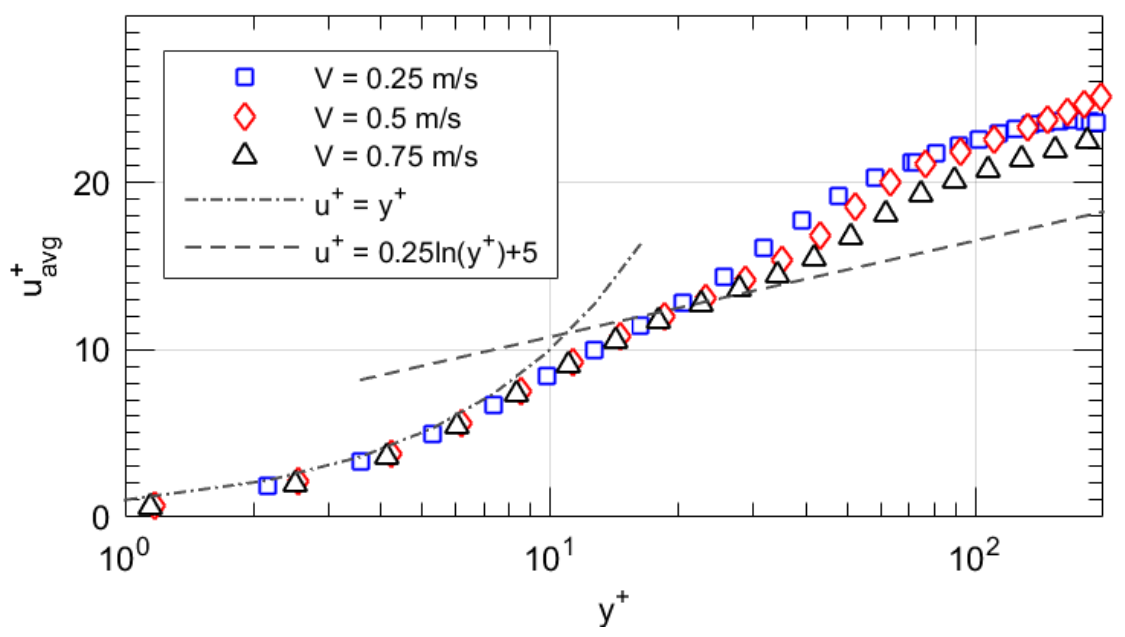

Fig. 7: Perfil de velocidad en la capa límite, numérico (con marcadores), ley de pared y ley de capa logarítmica

La Fig. 8 muestra la fluctuación de la presión estática en el tiempo del punto superior de la sección transversal del dominio fluidodinámico. Se observa que la amplitud de la presión incrementa con la velocidad del flujo. Dicha amplitud se puede caracterizar a través del parámetro estadístico RMS (valor cuadrático medio). Así, para la velocidad de $0.25 \mathrm{~m} / \mathrm{s}$ se tiene un RMS de $0.6857 \mathrm{~Pa}$; para la velocidad $0.5 \mathrm{~m} / \mathrm{s}, 4.9773 \mathrm{~Pa}$; y para la velocidad de $0.75 \mathrm{~m} / \mathrm{s}, 8.7303 \mathrm{~Pa}$. Este comportamiento se debe a que, al aumentar la velocidad de flujo, se incrementa la intensidad de la turbulencia y, con ello, el tamaño de los vórtices turbulentos. A medida que aumenta el tamaño de los vórtices, aumenta la disipación de energía y las fluctuaciones de presión en la pared. Esta tendencia concuerda con lo reportado por Bakewell et al. (1962), El Koury et al. (2013), y Pittard y Blotter (2003).
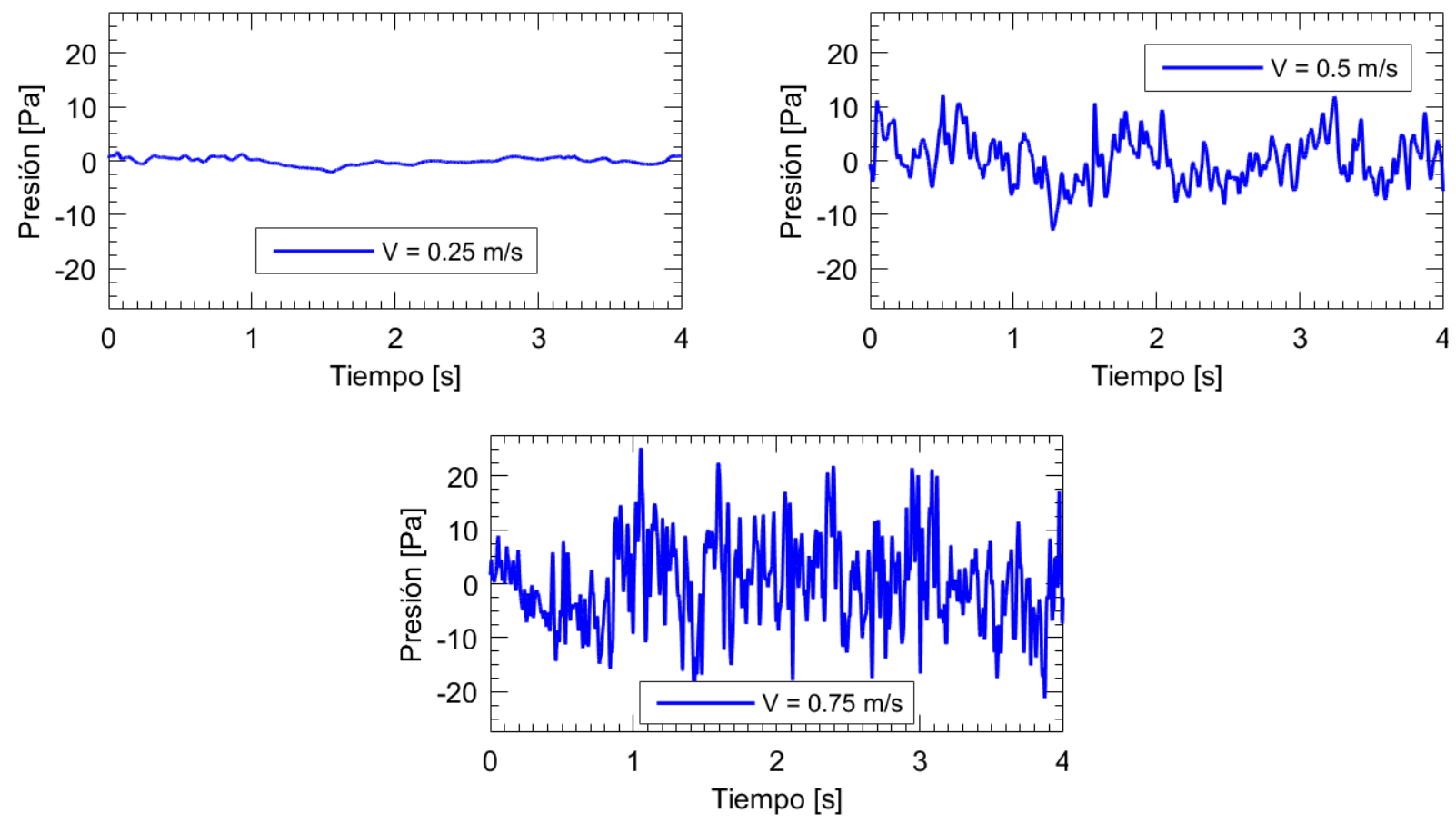

Fig. 8: Fluctuación de la presión en el punto superior del dominio del fluido

Los valores de la presión en el tiempo fueron transformados al dominio de la frecuencia. La Fig. 9 muestra los resultados expresados en PSD (espectro de densidad de potencia). Se observa que la energía de la presión (área bajo la curva) se incrementa con el aumento de la velocidad. Esto se debe al incremento de la turbulencia y su disipación de energía. Debe notarse que el PSD de la presión no contiene frecuencias picos, sino más bien es una señal que contiene un amplio espectro de frecuencias. Esto es característico de la turbulencia en fluidos (Silveira Neto, 2002). 

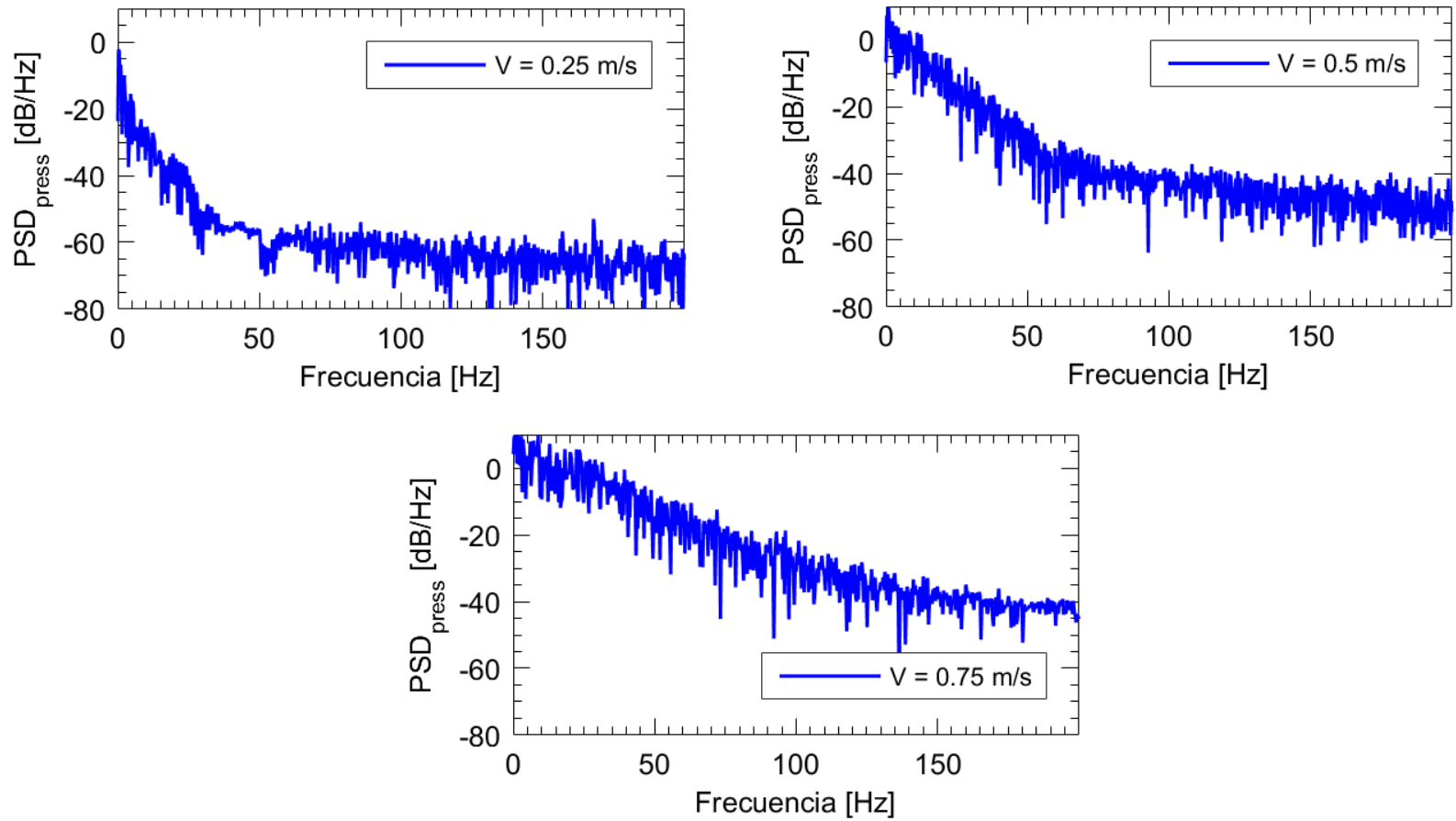

Fig. 9: Espectro de densidad de potencia (PSD) de la fluctuación de presión en el punto superior del dominio del fluido

\section{Comportamiento estructural}

La respuesta en el tiempo del desplazamiento del punto superior de la sección transversal de la tubería se muestra en la Fig. 10. Se observa que la amplitud del desplazamiento se incrementa con la velocidad del flujo. Dicha amplitud se puede caracterizar a través del parámetro estadístico RMS. Para la velocidad de $0.25 \mathrm{~m} / \mathrm{s}$ se tiene un valor RMS de $3.89 \times 10^{-6} \mathrm{~mm}$; para la velocidad de $0.5 \mathrm{~m} / \mathrm{s}, 39.04 \times 10^{-6} \mathrm{~mm}$; y para la velocidad de $0.75 \mathrm{~m} / \mathrm{s}, 46.94 \times 10^{-6} \mathrm{~mm}$. El incremento de la amplitud de desplazamiento con la velocidad está directamente relacionado con la fluctuación de la presión, que excita a la tubería. Esta tendencia concuerda con lo reportado por Evans et al. (2004), Ortiz-Vidal et al. (2013) y Ortiz-Vidal et al. (2017).
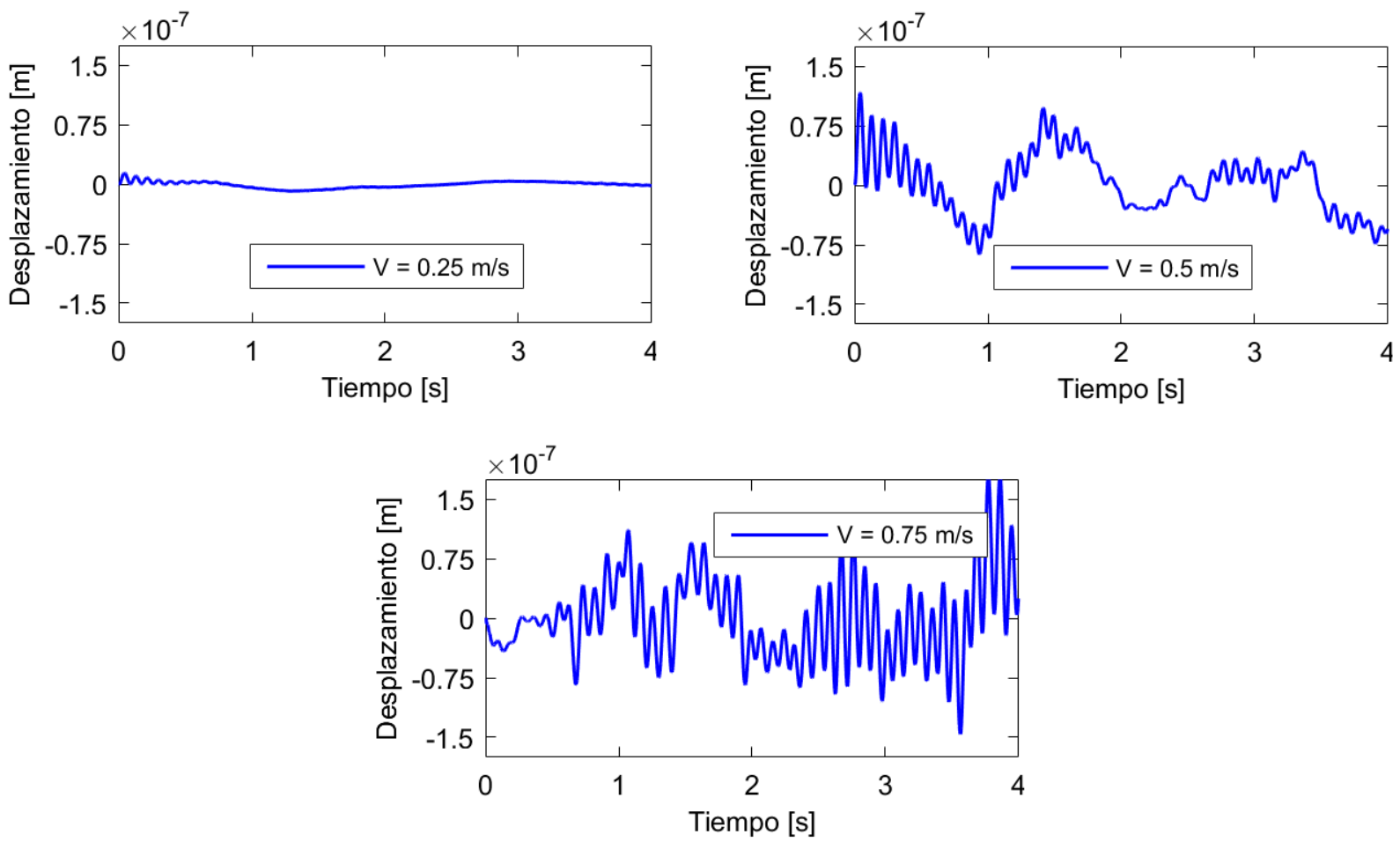

Fig. 10: Desplazamiento del punto superior del dominio estructural

Una respuesta transitoria en el desplazamiento estructural se refleja al inicio de la simulación, ver Fig. 10. Luego de ese intervalo inicial solo permanece la respuesta estacionaria. Ese transitorio puede deberse a que 
al inicio de la simulación acoplada la tubería está en reposo y de manera súbita se le impone el peso y las cargas del flujo turbulento totalmente desarrollado. Las respuestas transitorias de los casos con velocidad intermedia y alta muestran un comportamiento distinto. Por un lado, para $\mathrm{v}=0.5 \mathrm{~m} / \mathrm{s}$, la respuesta transitoria se muestra relevante obteniéndose órdenes de magnitud similares a la respuesta estacionaria. Por otro lado, para $v=0.75 \mathrm{~m} / \mathrm{s}$, la respuesta transitoria es despreciable cuando se compara con la respuesta estacionaria. Este comportamiento distinto puede deberse a la diferencia de velocidades de los flujos o al hecho de que el acoplamiento se realiza en un instante de tiempo cualquiera, donde las condiciones en cada instante son diferentes.

Las señales de desplazamiento en el dominio del tiempo fueron convertidas a aceleración y luego transformadas al dominio de la frecuencia. Los resultados en términos de PSD se muestran en la Fig. 11. Se observa que existe un incremento de la energía contenida en función de la velocidad del flujo. Estos resultados están de acuerdo con resultados experimentales reportados por Evans et al. (2004) y Ortiz-Vidal et al. (2017). El aumento de la amplitud en función de la velocidad está directamente relacionado con la respuesta en frecuencia de la presión (ver Fig. 9). Para mayores velocidades de flujo, la amplitud de la presión se intensifica en un mismo rango de frecuencia. Entonces, es de esperarse un incremento de la magnitud del PSD de la aceleración, pues es la presión la carga que excita a la tubería. Esto se cumple principalmente para las frecuencias más bajas, aunque se observa poca diferencia entre las magnitudes de los casos con velocidad intermedia $(0.5 \mathrm{~m} / \mathrm{s})$ y alta $(0.75 \mathrm{~m} / \mathrm{s})$.

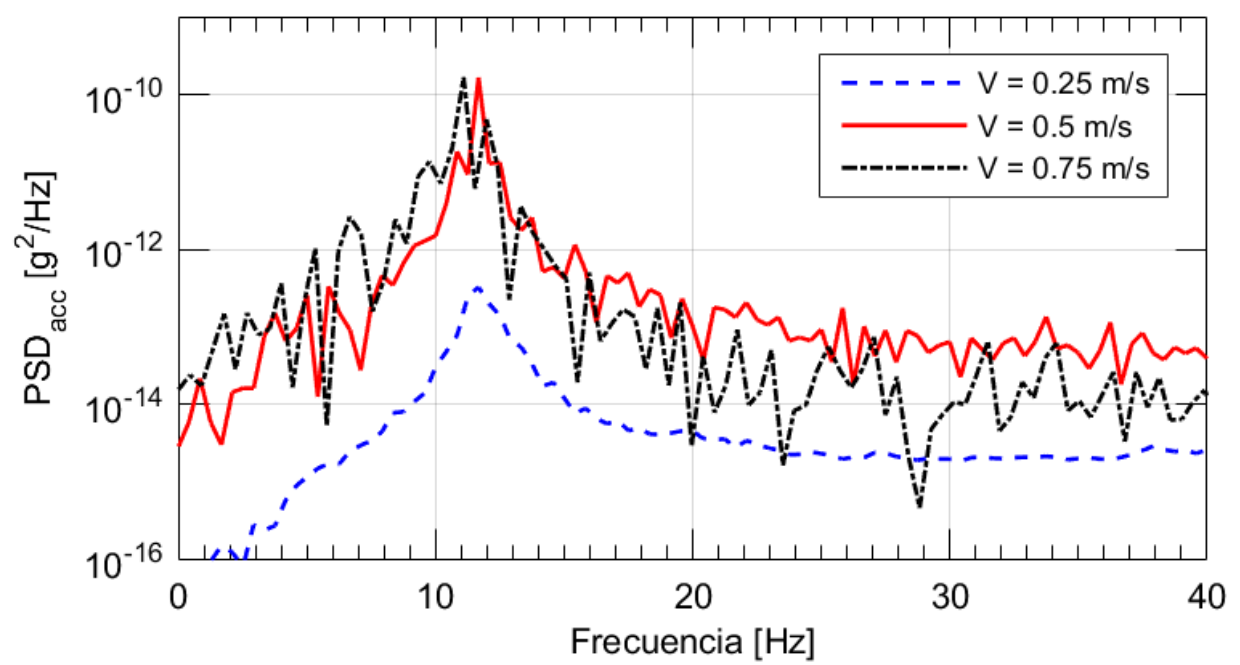

Fig. 11: Espectro de densidad de potencia (PSD) de la aceleración del punto superior del dominio estructural

Estudios experimentales y analíticos (Evans et al., 2004; Ortiz-Vidal et al., 2017; Païdoussis, 1998), han reportado que la frecuencia pico de vibración disminuye conforme se incrementa la velocidad del flujo. En la Fig. 11 puede observarse el comportamiento de la frecuencia pico del sistema flujo-tubería estudiado. Los resultados muestran que la frecuencia disminuye ligeramente a medida que aumenta la velocidad. Por ejemplo, para $0.25 \mathrm{~m} / \mathrm{s}$, la frecuencia pico es $11.86 \mathrm{~Hz}$; para $0.5 \mathrm{~m} / \mathrm{s}, 11.78 \mathrm{~Hz}$; y para $0.75 \mathrm{~m} / \mathrm{s}, 11.26 \mathrm{~Hz}$.

\section{CONCLUSIONES}

Se presenta un procedimiento para el análisis del fenómeno de vibración inducida por flujo turbulento en tubería usando simulación numérica. El procedimiento consta de un modelo numérico y la estrategia de simulación correspondiente. Se trata de un modelo tridimensional, con enfoque de Interacción FluidoEstructura y acoplamiento bidireccional. Los puntos a destacar de la estrategia de simulación consisten en la técnica para la discretización espacial y temporal, la condición de periodicidad para el flujo, el modelo WMLES S-Omega para la turbulencia y la teoría de grandes desplazamientos para la tubería.

Los resultados muestran que el procedimiento captura la física del fenómeno de vibración inducida por flujo. La fluctuación de la presión actúa en un amplio espectro de frecuencias y su intensidad se incrementa con la velocidad del flujo. Con relación a la respuesta estructural, la amplitud de la vibración se incrementa con el aumento de la velocidad del flujo. Contrariamente, la frecuencia pico disminuye con el incremento de esta velocidad. De esta forma, el presente procedimiento se muestra adecuado para analizar el fenómeno de vibración inducida por flujo monofásico turbulento en tuberías. 


\section{AGRADECIMIENTOS}

A la Dirección de Gestión de la Investigación de la Pontificia Universidad Católica del Perú, por el financiamiento del proyecto "ID 576 - Análisis numérico y validación experimental de la vibración inducida por flujo en una tubería horizontal que transporta flujo bifásico agua - aire", que fue la base para el desarrollo de la presente investigación.

\section{REFERENCIAS}

ANSYS, Inc., ANSYS Academic Research, Release 19.0, Help System, (2018).

Ashley, H. y Haviland, G., Bending Vibration of a Pipe Line Containing Flowing Fluid, Journal of Applied Mechanics, 17(3), 229-232 (1950).

Bakewell, H.P., Carey, G.F., Libuha, J.J., Schloemer, H.H. y Von Winkle, W.A., Wall pressure correlations in turbulent pipe flow, U.S. Navy Underwater Sound Laboratory, No. NUSL-559 (1962).

Cengel, Y.A. y Cimbala, J.M., Internal Flow, in Fluid Mechanics: Fundamentals and Applications, $4^{\text {th }}$ Ed., McGraw-Hill Education, pp 351-442, New York, USA (2018).

Eggels, J. G. M., Direct and Large Eddy Simulation of Turbulent Flow in a Cylindrical Pipe Geometry, Doctoral Thesis, Faculty Mechanical Maritime and Material Engineering, Delft University of Technology-Netherlands (1994).

El Khoury, G.K., Schlatter, P. y otros cuatro autores, Direct numerical simulation of turbulent pipe flow at moderately high Reynolds numbers, doi: 10.1007/s10494-013-9482-8, Flow Turbulence Combust, 91(3), 475-495 (2013).

Evans, R.P., Blotter, J.D. y Stephens, A.G., Flow Rate Measurements Using Flow-Induced Pipe Vibration, doi:10.1115/1.1667882, ASME Journal Fluids Engineering, 126(2), 280-285 (2004).

Fernández O, J.M., Algunas ideas fundamentales; en Técnicas numéricas en Ingeniería de Fluidos, pp 27-46, Ed. Reverté, Barcelona, España (2012).

Ferziger, J.H. y Peric, M., Methods for Unsteady Problems; in Computational Methods for Fluid Dynamics, $3^{\text {rd }}$ Ed., Springer, pp 135-156, New York, USA (2002).

Fox, R.W., McDonald, A.T. y Pritchard, P.J., Part B. Flow in pipes and ducts. Internal incompressible viscous flow; in Introduction to Fluid Mechanics, $9^{\text {th }}$ Ed., Wiley, pp 292-325, USA (2015).

Hernandez-Perez, V., Abdulkadir, M. y Azzopardi, B.J., Grid Generation Issues in the CFD Modelling of Two-Phase Flow in a Pipe, doi: 10.1260/1757-482X.3.1.13, The Journal of Computational Multiphase Flows, 3(1), 13-26 (2011).

Liu, B., Yan, Q. y Wei, W., Numerical investigations of the flow induced oscillation of a torque converter, doi: 10.1080/19942060.2017.1419149, Journal Engineering Applications of Computational Fluid Mechanics, 12(1), 270-281 (2018).

Loh, S.K., Faris, W.F. y Hamdi, M., Fluid-structure interaction simulation of transient turbulent flow in a curved tube with fixed supports using LES, doi: 10.1504/pcfd.2013.050646, Progress in Computational Fluid Dynamics, 13(1), 11-19 (2013).

Newmark, N.M., A Method of Computation for Structural Dynamics, Journal of the Engineering Mechanics Division, 85(3), 67-94 (1959).

Ortiz-Vidal, L.E., Cabanillas, D.E. y Fierro, R.A., Equilibrio hidráulico en sistemas de bombeo minero: Estudio de caso, doi: 10.4067/S0718-33052010000300007, Ingeniare. Revista chilena de ingeniería, 18(3), 335-342 (2010).

Ortiz-Vidal, L.E., Rodriguez, O.M. y Mureithi, N.W., An Exploratory Experimental Technique to Predict Two-Phase Flow Pattern from Vibration Response, doi: 10.115/PVP2013-98115, Proceeding of the ASME 2013 Pressure Vessels and Piping Conference. Volume 4: Fluid-Structure Interaction, American Society of Mechanical Engineers Digital Collection, Paris, France (2014).

Ortiz-Vidal, L.E., Mureithi, N.W. y Rodriguez, O.M., Vibration response of a pipe subjected to two-phase flow: Analytical formulations and experiments, doi: 10.1016/j.nucengdes.2016.12.020, Nuclear Engineering and Design, 313, 214-224 (2017).

Païdoussis, M.P., Fluid-structure interactions: slender structures and axial flow, Vol.1, pp 1-573, Academic Press, California, USA (1998).

Park, G.I. y Moin, P., Space-time characteristics of wall-pressure and wall shear-stress fluctuations in wall-modeled large eddy simulation, doi: 10.1103/PhysRevFluids.1.024404, Physical Review Fluids, 1(2), 024404 (2016).

Pei, J., Dohmen, H., Yuan, S. y Benra, F., Investigation of unsteady flow-induced impeller oscillations of a single-blade pump under off-design conditions, doi: 10.1016/j.jfluidstructs.2012.08.005, Journal of Fluids and Structures, 35, 89-104 (2012).

Peng, C., Geneva, N., Guo, Z. y Wang, L., Direct numerical simulation of turbulent pipe flow using the lattice Boltzmann method, doi: 10.1016/j.jcp.2017.11.040, Journal of Computational Physics, 357, 16-42 (2018). 
Piomelli, U. Large-Eddy Simulation: Where We Stand, ISBN: 1570743657, $1^{\text {st }}$ International conference, Advances in DNS/LES: Direct numerical simulation and large eddy simulation, Ruston, LA, USA, 93-104 (1997).

Pittard, M.T. y Blotter, J.D., Numerical modeling of LES based turbulent flow induced vibration, doi: 10.1115/IMECE200342541, Proceedings of the ASME 2013 International Mechanical Engineering Congress and Exposition. Noise Control and Acoustics Division, American Society of Mechanical Engineers Digital Collection, Washington, DC, USA, 141-148 (2003).

Sayadi, T. y Moin, P., Large eddy simulation of controlled transition to turbulence, doi: 10.1063/1.4767537, Physics of Fluids, 24, 114103 (2012).

Shurtz, T., Maynes, D. y Blotter, J., Analysis of induced vibrations in fully-developed turbulent pipe flow using a coupled LES and FEA approach, doi: 10.1115/FEDSM-ICNMM2010-30477, Proceedings of the ASME $20103^{\text {rd }}$ Joint US-European Fluids Engineering Summer Meeting, Montreal, Quebec, Canada, 521-531 (2010).

Silveira Neto, A., Fundamentos da turbulência nos fluidos, Associação Brasileira de Ciências Mecânicas (ABCM), 1, 1-52 (2002).

Wagner, C., Huttl, T.J. y Friedrich, R., Low-Reynolds-number effects derived from direct numerical simulation in turbulent pipe flow, doi: 10.1016/S0045-7930(01)00007-X, Journal of Fluid Mechanics, 608, 81-112 (2008).

White, F.M., Fluid Mechanics, $7^{\text {th }}$ Ed., McGraw Hill, New York, USA (2011).

Wu, X. y Moin, P., A direct numerical simulation study on the mean velocity characteristics in turbulent pipe flow, doi: 10.1017/S0022112008002085, Journal of Fluid Mechanics, 608, 81-112 (2008).

Zienkiewicz, O.C., Taylor, R.L. y Nithiarasu, P., The Finite Element Method for Fluid Dynamics, $7^{\text {th }}$ Ed., ButterworthHeinemann, Boston, USA (2014). 